\title{
Time-Varying Feedback Laws for Decentralized Control
}

\author{
BRIAN D. O. ANDERSON, FELLOW, IEEE, AND JOHN B. MOORE, FELLOW, IEEE
}

\begin{abstract}
Decentralized control schemes are considered for timeinvariant, finite dimensional, linear systems with known state equations. It is assumed that the systems are reachable and observable at a fictitious centralized control station, and that there is strong connectivity between the decentralized control stations via the system where necessary. It is shown that whether or not there are decentralized fixed modes in the open-loop system, periodically varying feedback gains at all but one of the control stations permit the remaining control station to observe and control the system given knowledge of the control laws implemented at the other control stations.

Certain time-invariant systems which cannot be stabilized by decentralized time-invariant controllers, namely those with unstable decentralized fixed modes, can thus be stabilized by decentralized time-varying controllers.
\end{abstract}

\section{INTRODUCTION}

$\mathrm{C}$ ONSIDER the time invariant, finite-dimensional, linear, $s$-channel decentralized control system

$$
\begin{gathered}
\dot{x}=A x(t)+\sum_{i=1}^{s} B_{i} u_{i}(t) \\
y_{i}(t)=C_{i} x(t) \quad i=1,2, \cdots, s
\end{gathered}
$$

with states $x(\cdot) \in \boldsymbol{R}^{n}$, inputs $u_{i}(\cdot) \in \boldsymbol{R}^{m_{1}}$ and outputs $y_{i}(\cdot) \in$ $\boldsymbol{R}^{p_{i}}$. The $i$ th control station is assumed to have access only to the past measurements $\left\{y_{i}(\cdot)\right\}$ and past controls $\left\{u_{i}(\cdot)\right\}$, and the control laws implemented at the other control stations.

To avoid trivial situations, we assume that the system is completely controllable and observable at a fictitious centralized control and measurement station, i.e., $\left\{A,\left[B_{1}\right.\right.$

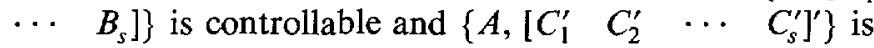
observable. We term this centralized controllability and observability. Moreover, to obtain an interesting problem it is generally assumed that the system is not both completely controllable and observable on any one of the $s$ channels.

As noted in [1], such decentralized systems are useful models for power systems with each control and measurement being associated with a power station, or for economic systems where, for example, each control and measurement station is associated with a government department. In such systems, the implementation of a centralized

\footnotetext{
Manuscript received March 27, 1980; revised December 15, 1980. This work was supported by the Australian Research Grants Committee.

The authors are with the Department of Electrical Engineering, University of Newcastle, Newcastle, N.S.W., Australia.
}

control station with access to all controls and measurements may be prohibitively complex.

For simplicity of implementation, it may often be acceptable to achieve control via a time-invariant output feedback law on all but one channel

$$
u_{i}(t)=K_{i} y_{i}(t)
$$

so as to achieve reachability and observability at the remaining control/measurement station. (Then standard state estimation and control techniques can be applied to achieve pole assignability or at least some suitable control of the system.) The theory of [2], [3], building on [4], [5], for such control systems exposes two requirements for this capability. First, the system must have a connectivity property termed strong connectivity. Roughly, if arbitrary feedbacks of the form (1.2) are employed, then between control station $i$ and measurement station $j$ for every $i \neq j$, there must be (after feedback) a nonzero transfer function matrix; for more details, see [2], [3] and the Appendix. Second, there must be no fixed modes, i.e., if feedback laws of the form (1.2) are implemented for every $i$, then the closedloop system matrix, $A+\sum_{i} B_{i} K_{i} C_{i}$ must not have any eigenvalues which are independent of the $K_{i}$.

The fixed modes associated with a decentralized control system arise when, as is commonly the case, there are patterns in elements of the system matrices, such as when certain elements are equal or are simply multiples of one another, or are zero. A simple rank test to detect decentralized fixed modes is given in [6]. This reference also clarifies the connection between the definition of decentralized fixed modes in [4], [5] and their appearance in the decentralized control problem of [2], [3].

Decentralized fixed modes are a generalization of a familiar concept in centralized control. If the system $\dot{x}=A x$ $+B u, y=C x$ has the property that among the closed-loop eigenvalues of $A+B K C$, associated with use of the control $u=K y$, there are one or more which are independent of $K$, then such eigenvalues are termed (centralized) fixed modes such fixed modes are present if and only if there is failure of one of both of complete controllability and observability. As a consequence, no matter what control law is used-linear or nonlinear, dynamic or nondynamic, distributed or finite-dimensional - the fixed mode will still be present in the sense that if $\lambda_{i}$ is such a mode, the closed-loop 
response for a suitable initial condition will contain terms proportional to $\exp \lambda_{i} t$.

The question arises as to whether decentralized fixed modes remain when controllers other than those of the form (1.2) are used. By analogy with the centralized case, one might expect so, and indeed in [5] it is shown that when the controllers defined in (1.2) are replaced by arbitrary linear, time-invariant, finite-dimensional controllers, the fixed modes remain. In [6], the finite-dimensionality constraint is removed. Despite this parallel with the centralized fixed mode ideas however, the analogy has a definite limitation. For it turns out that more general controllers

$$
u_{i}(t)=F\left\{y_{i}(\tau), u_{i}(\tau), \tau \in[0, t]\right\}
$$

can be used to eliminate the fixed modes as pointed out in [7], in the sense that such decentralized controllers for a particular class of systems with fixed modes can bring an arbitrary initial state to the zero state by an open-loop control approach.

This observation then raises the issue of whether a decentralized feedback controller could be designed, even in the presence of decentralized fixed modes. Such a controller must sacrifice one of the properties of linearity and time-invariance. We choose to sacrifice the latter. Our first result is for two channel systems: if $u_{2}(t)=K_{2}(t) y_{2}(t)$ where $K_{2}(t)$ is periodic and piecewise constant, taking $\rho \geqslant 1+\max \left(\operatorname{dim} u_{2}, \operatorname{dim} y_{2}\right.$ ) values, then strong connectivity [3], even when a fixed mode is present, is enough to ensure that the system is uniformly controllable from $u_{1}$ and uniformly observable from $y_{1}$. (Centralized controllability and observability are of course assumed.) Thus, fixed modes only present a problem when there is a restriction to time-invariant controllers.

Put another way, the results of this paper show that time-varying controllers may be necessary to control certain time-invariant systems, namely those with fixed modes. Moreover, to achieve satisfactory control in systems close in some sense to ones with fixed modes, there could well be advantages in employing time-varying controllers.

In the next section, the results for the two channel case are derived. These are then generalized in section three to the multichannel situation.

\section{The Case of Two Control Systems}

Consider the decentralized system with two control and measurement stations with associated matrices $\left\{A,\left[B_{1}\right.\right.$ $\left.\left.B_{2}\right],\left[\begin{array}{ll}C_{1}^{\prime} & C_{2}^{\prime}\end{array}\right]^{\prime}\right\}$. Suppose also that the second station implements the control law $u_{2}(t)=K_{2}(t) y_{2}(t)$. Then the system viewed from the first control and measurement stations can be represented by the triple $\{A+$ $\left.B_{2} K_{2}(t) C_{2}, B_{1}, C_{1}\right\}$. In this section, we seek conditions for the uniform controllability and observability of this triple. Satisfaction of these conditions means that we can design an observer/linear state feedback pair, possibly by linearquadratic optimization, which will stabilize the system.
Let $\phi_{K_{2}}(t, s)$ denote the transition matrix associated with $A+B_{2} K_{2}(t) C_{2}$. We denote the observability Grammian by

$$
W_{s+T, s}=\int_{s}^{s+T} \phi_{K_{2}}^{\prime}(t, s) C_{1}^{\prime} C_{1} \phi_{K_{2}}(t, s) d t .
$$

In case $\left[A, C_{1}\right]$ is observable, it is trivial to secure the desired observability - one simply takes $K_{2}(t) \equiv 0$. In the main, we shall therefore concentrate on the case when $\left[A, C_{1}\right]$ is not observable. Then in order to achieve observability of the pair $\left[A+B_{2} K_{2}(t) C_{2}, C_{1}\right]$, it makes sense, as we shall argue, to make two assumptions.

\section{Centralized Observability Assumption:}

$$
\left\{A,\left[\begin{array}{ll}
C_{1}^{\prime} & C_{2}^{\prime}
\end{array}\right]^{\prime}\right\} \text { is observable. }
$$

\section{Connectivity Assumption:}

$$
C_{1}(s I-A)^{-1} B_{2} \not 0 \text {. }
$$

If the first assumption fails, then for all $K_{2}(t),\{A+$ $\left.B_{2} K_{2}(t) C_{2},\left[\begin{array}{ll}C_{1}^{\prime} & C_{2}^{\prime}\end{array}\right]^{\prime}\right\}$ is unobservable and so, a fortiori, is $\left\{A+B_{2} K_{2}(t) C_{2}, C_{1}\right\}$.

The second assumption (2.3) is only important in case $\left[A, C_{1}\right]$ is not observable. To see its importance, we argue first algebraically, and then heuristically. By noting that $\phi_{K_{2}}(t, s)$ is the solution of

$$
\dot{X}=A X+B U
$$

where $X(s)=I, U(t)=K_{2}(t) C_{2}(t) \dot{\phi}_{K_{2}}(t, s)$, we see that

$$
\phi_{K_{2}}(t, s)=e^{A(t-s)}+\int_{s}^{t} e^{A(t-\tau)} B_{2} K_{2}(\tau) C_{2} \phi_{K_{2}}(\tau, s) d \tau .
$$

If (2.3) fails, i.e., if $C_{1} e^{A t} B_{2} \equiv 0$, we see from (2.4) that

$$
C_{1} \phi_{K_{2}}(t, s)=C_{1} e^{A(t-s)} \text {. }
$$

The observability Grammian (2.1) becomes the same as that associated with $\left[A, C_{1}\right]$ and if this pair is unobservable, (2.1) cannot then be nonsingular.

The need for (2.3) is also in accord with intuition. If $\left[A, C_{1}\right]$ is not observable, observation station one needs to find out something about what observation station two observes, as well as to use its own direct observation to deduce the state. The idea is that some of what observation station two observes, viz. $C_{2} x$, is fed back to control station two as $K_{2}(t) C_{2} x$, and shows up at observation station one through the nonzero transmission path with transfer function matrix $C_{1}(s I-A)^{-1} B_{2}$. In this way, observation station one acquires information "originally" possessed only by observation station two.

We now state the following.

Lemma 2.1: With notation as above and assumptions (2.2) and (2.3) in force, suppose $K_{2}(t) \equiv 0$ in $\left[s, s_{1}\right)$ and $K_{2}(t) \equiv K_{2} \not \geq 0$ in $\left[s_{1}, s+T\right)$ for arbitrary $s_{1} \in(s, s+T)$. Suppose further that $u_{2}, y_{2}$ are scalar. Then $W_{s+\tau, s}$ is nonsingular. 
Proof (Case 1): $C_{1}(s I-A)^{-1} B_{2}$ is a scalar transfer function. Suppose that $W_{s+T, s} \gamma=0$ for some $\gamma \neq 0$. We shall deduce a contradiction; in fact we shall show that $C_{1} A^{i} \gamma=0, C_{2} A^{i} \gamma=0$ for all $\gamma$, contradicting (2.2). Now use of (2.1) yields

$$
\begin{aligned}
C_{1} e^{A(t-s)} \gamma=0 & & t \in\left[s, s_{1}\right] \\
C_{1} e^{\left(A+B_{2} K_{2} C_{2}\right)\left(t-s_{1}\right)} e^{A s_{1}} \gamma=0 & & t \in\left[s_{1}, s+T\right]
\end{aligned}
$$

or, with $\delta=e^{A s_{1}} \gamma$,

$$
\begin{aligned}
C_{1} A^{i} \delta & =0 \\
C_{1}\left(A+B_{2} K_{2} C_{2}\right)^{i} \delta & =0
\end{aligned}
$$

for all integer $i$. Let $q$ be the least nonnegative integer for which $C_{1} A^{q} B_{2} \neq 0$, existing by (2.3). This definition and (2.6a) imply that (2.6b) holds trivially for $i<q$. Writing (2.6b) for $i=q, q+1, \cdots$ with the aid of (2.6a) gives

$$
\left[\begin{array}{cccc}
C_{1} A^{q} B_{2} K_{2} & 0 & 0 \\
* & C_{1} A^{q} B_{2} K_{2} & 0 & \\
* & * & C_{1} A^{q} B_{2} K_{2} & \\
* & * & * & \ddots \\
* & * & * &
\end{array}\right]\left[\begin{array}{c}
C_{2} \\
C_{2} A \\
C_{2} A^{2} \\
\vdots
\end{array}\right] \delta=0 .
$$

(Entries below the diagonal are irrelevant.)

Now (2.7) implies that $C_{2} A^{i} \delta=0$ for all $i$. With $C_{1} A^{i} \delta=0$ for all $i$, the complete observability of $\left\{A,\left[\begin{array}{ll}C_{1}^{\prime} & C_{2}^{\prime}\end{array}\right]^{\prime}\right\}$ is contradicted.

Case 2: $C_{1}(s I-A)^{-1} B_{2}$ is a vector of transfer functions. Let $C_{1}^{j}$ denote the $j$ th row of $C_{1}$ and set

$$
W_{s+T, s}^{j}=\int_{s}^{s+T} \phi_{K_{2}}^{\prime}(t, s)\left(C_{1}^{j}\right)^{\prime} C_{i}^{j} \phi_{K_{2}}(t, s) d t .
$$

Then

$$
W_{s+T, s}=\sum_{j} W_{s+T, s}^{j}
$$

Suppose $W_{s+T, s} \gamma=0$. Then $W_{s+T, s}^{j} \gamma=0$ for each $j$. The Case 1 argument yields that $C_{1}^{j} A^{i} \delta=0$ and $C_{2} A^{i} \delta=0$ for all $i$ and those $j$ for which $C_{1}^{j}(s I-A)^{-1} B_{2} \neq 0$ (The set of such $j$ is nonempty by assumption.) Also, if $j$ is such that $C_{1}^{j}(s I-A)^{-1} B_{2} \equiv 0$, we know that $C_{1}^{j} \phi_{K_{2}}(t, s)=C_{1}^{j} e^{A(t-s)}$ and so we get $C_{1}^{j} A^{i} \delta=0$ for all $i$. Thus, for all $j, C_{1}^{j} A^{i} \delta=0$, i.e., $C_{1} A^{i} \delta=0$, for all $i$. Since also $C_{2} A^{i} \delta=0$, we obtain the desired contradiction.

$\nabla \nabla \nabla$

In Lemma 2.1, $u_{2}$ and $y_{2}$ were restricted to being scalars. We now remove this restriction. The idea is to use $K_{2}(t)$ to switch each of the components of the second output station one at a time into a feedback to any component of the second input station which is connected via the system to the first output station.

Lemma 2.2: With notation as above and assumptions (2.2) and (2.3) in force, suppose with $B_{2}^{j}$ the $j$ th column of $B_{2}$, one has $C_{1}(s I-A)^{-1} B_{2}^{j} \not 0$. Suppose further that with $e_{i}$ denoting the unit vector of appropriate dimension with 1 in the $i$ th position and with $p_{2}$ the dimension of $y_{2}$, $K_{2}(t) \equiv 0$ for $t \in\left[s, s_{1}\right), K_{2}(t) \equiv k_{2}^{1} e_{j} e_{1}^{\prime}$ for $t \in\left[s, s_{1}\right), \cdots$ $K_{2}(t)=k_{2}^{p^{2}} e_{j} e_{p_{2}}^{\prime}$ for $t \in\left[s_{p_{2}-1}, s+T\right)$, where the $k_{2}^{j}$ are nonzero constants and $s<s_{1}<\cdots<s_{p_{2}-1}<s+T$. Then $W_{s+T, s}$ is nonsingular.

Proof: For convenience, suppose $C_{1}$ has one row only (The Case 2 argument of Lemma 2.1 can be used otherwise). Then $W_{s+T, s} \gamma=0$ implies

$$
\begin{array}{rr}
C_{1} e^{A(t-s)} \gamma \equiv 0 & \text { for } t \in\left[s_{0}, s_{1}\right] \\
C_{1} e^{\left(A+B_{2}^{j} k_{2}^{1} C_{2}^{1}\right)\left(t-s_{1}\right) e^{A s_{1}} \gamma \equiv 0} & \text { for } t \in\left[s_{1}, s_{2}\right] \\
C_{1} e^{\left(A+B_{2}^{j} k_{2}^{2} C_{2}^{2}\right)\left(t-s_{2}\right)} e^{\left(A+B_{2}^{j} k_{2}^{1} C_{2}^{1}\right)\left(s_{2}-s_{1}\right)} e^{A s} \gamma \equiv 0 & \text { for } t \in\left[s_{2}, s_{3}\right],
\end{array}
$$

etc. Arguing as in the proof of Lemma 2.1, the first two identities imply that

$$
C_{1} A^{i} \delta_{1}=0 \quad C_{2}^{1} A^{i} \delta_{1}=0
$$

for all $i$ and $\delta_{1}=e^{A s_{1}} \gamma$. Set $\delta_{2}=e^{\left(A+B_{2}^{j} k_{2}^{1} C_{2}^{1}\right)\left(s_{2}-s_{1}\right)} \delta_{1}$. It is trivial to conclude that $C_{1} A^{i} \delta_{2}=0, C_{2}^{1} A^{i} \delta_{2}=0$ for all $i$. The first of these identities taken with $(2.8 \mathrm{c})$ yields $C_{2}^{2} A^{i} \delta_{2}=0$ for all $i$. Proceeding in this fashion, we construct a vector $\delta_{p_{2}}$ for which $C_{1} A^{i} \delta_{p_{2}}=0$ and $C_{2}^{j} A^{i} \delta_{p_{2}}$ for all $i$ and $j$. This violates the observability Assumption (2.2). $\quad \nabla \nabla \nabla$

Examination of the above argument will show that if a matrix $\bar{C}_{2}$ obtained from $C_{2}$ by eliminating certain rows has the property that $\left\{A,\left[\begin{array}{ll}C_{1}^{\prime} & \bar{C}_{2}^{\prime}\end{array}\right]^{\prime}\right\}$ is observable, then one can in effect avoid feedback of those entries of $y_{2}$ corresponding to the rows of $C_{2}$ omitted in forming $\bar{C}_{2}$, thereby allowing $K_{2}(t)$ to take fewer values.

Reviewing to this point, we know that to make $W_{s+T, s}$ nonsingular, it is enough

a) to have $\left[A, C_{1}\right]$ observable, for then $K_{2}(t) \equiv 0$ works,

b) if $\left[A, C_{1}\right]$ is not observable, to have centralized observability (2.2) and connectivity (2.3) and absence of a fixed modes in the sense that [6]

$$
\operatorname{rank}\left[\begin{array}{cc}
\lambda I-A & B_{2} \\
C_{1} & 0
\end{array}\right] \geqslant_{n}
$$

for all complex $\lambda$, for then, as shown in [2], [8], almost all constant $K_{2}$ will suffice,

c) if $\left[A, C_{1}\right]$ is not observable, to have centralized observability (2.2) and connectivity (2.3). Then a $K_{2}(t)$ taking $p_{2}+1$ piecewise constant values in $[s, s+T]$ suffices.

Now choosing a $K_{2}(t)$ for $t \in(-\infty, \infty)$ is easy. We simply choose $K_{2}(t)$ to be periodic with period $T$. If $W_{s+r, s}$ is nonsingular, it is a standard result that the pair $\left[A+B_{2} K_{2}(t) C_{2}, C_{1}\right]$ is uniformly observable (see [9]).

It is not hard to see that if $K_{2}(t)$ takes any $\rho$ differing piecewise constant values for any finite $\rho>p_{2}+1$, and is periodic, this result still holds. Lack of observability is characterized by the satisfaction of certain multivariable equalities in the entries of $K$ at each of its set values. We have shown via Lemma 2.2 that these equalities need not be satisfied for one particular set of values (those where $K$ is zero over one interval and where certain equalities 
among the $\rho$ values exist so that in fact only $p_{2}+1$ are different). Therefore, for almost all choices of $K$, the equalities will fail. This is a typical argument of algebraic geometry; for an introduction to these ideas (see, e.g., [10]).

The dual result for controllability is easy to obtain. Obviously, we require the following.

\section{Centralized Controllability Assumption:}

$$
\left\{A,\left[B_{1}, B_{2}\right]\right\} \text { is controllable. }
$$

\section{Connectivity Assumption:}

$$
C_{2}(s I-A)^{-1} B_{1} \neq 0 \text {. }
$$

(The intuitive idea behind $(2.11)$ is that it permits the feeding in of signals at control station one which couple through to output station two, and thus via feedback to input station two, so as to affect states normally accessible only from input station two; the assumption is unnecessary if $\left[A, B_{1}\right]$ is completely controllable.)

In summary, we have proved the following.

Theorem 2.1: Consider the decentralized control systems (1.1) for the case of two control and output stations and assume it is controllable and observable in the centralized sense. Consider periodic feedback gains $K_{2}(t)$ from output station two to input station two, with arbitrary period $T$. Then $\left[A+B_{2} K_{2}(t) C_{2}, B_{1}\right]$ is uniformly controllable if the connectivity Assumption (2.11) holds and $K_{2}(t)$ is piecewise constant taking at least $m_{2}+1$ distinct values. Dual results holds for uniform observability.

We remark that similar results can be obtained for discrete time, save that as a result of the dichotomies between constructibility and observability between controllability and reachability which arise when singular transition matrices are possible, the result is one involving controllability and constructibility. Of course, this poses no problem for application: controllability and constructibility are what is needed.

Also, for completeness we remark that the alternative known conditions for the desired controllability of $[A+$ $\left.B_{2} K_{2}(t) C_{2}, B_{1}\right]$ are that $\left[A, B_{1}\right]$ is controllable (and then almost all constant $K_{2}$, including $K_{2}=0$ work) or that the connectivity assumption (2.11) holds and

$$
\operatorname{rank}\left[\begin{array}{cc}
\lambda I-A & B_{1} \\
C & 0
\end{array}\right] \leqslant n
$$

for all complex $\lambda$ (and then almost all constant $K_{2}$ work).

Example: A second-order nontrivial example with fixed modes and the required controllability, observability properties does not appear to exist. Consider the third-order example

$$
\begin{aligned}
& A=\left[\begin{array}{lll}
1 & 0 & 0 \\
0 & 1 & 0 \\
0 & 0 & 2
\end{array}\right] \\
& C_{1}^{\prime}=B_{2}=\left[\begin{array}{l}
0 \\
0 \\
1
\end{array}\right] \\
& B_{1}=C_{2}^{\prime}=\left[\begin{array}{ll}
0 & 1 \\
1 & 0 \\
0 & 0
\end{array}\right] .
\end{aligned}
$$

Then there is a fixed mode at $\lambda=1$ since

$$
\operatorname{rank}\left[\begin{array}{cc}
\lambda I-A & B_{2} \\
C_{1} & 0
\end{array}\right]=2<n=3 .
$$

Consider the closed-loop matrix $\left[A+B_{2} K_{2}(t) C_{2}\right]$ where $K_{2}(t)=\left[\begin{array}{ll}0 & 1\end{array}\right]$ for $t \in[2 k, 2 k+1)$, and $K_{2}(t)=\left[\begin{array}{ll}1 & 0\end{array}\right]$ for $t \in[2 k+1,2 k+2)$ for $k=0,1,2, \cdots$. Then the observability and controllability matrices (calculated analytically) over the range $[2 k, 2 k+2)$ for all $k=0,1, \cdots$ are positive definite with condition numbers approximately 100,6 , respectively. This first trial periodic control gain $K_{2}(t)$ achieves reasonable controllability and observability properties. Undoubtedly, a search procedure could provide an improvement if required. With the above first trial selection $K_{2}(\cdot)$, undoubtedly the observability properties are somewhat sensitive to small parameter variations in some of the entries of the system matrices; on the other hand, for the case when $K_{2}(\cdot)$ is a constant and is used on a perturbation of the given system, the observability properties will be highly sensitive to the perturbation. Thus, even if there is no decentralized fixed mode, it may be advisable to use periodically varying gains.

An interesting feature of the control/estimation scheme, shared with that applicable in case no decentralized fixed modes exist (see [2], [3]), is the asymmetry in the ultimate controller structure. One channel has time-varying memoryless feedback round it, while the other has an estimator/control-law combination. Spreading the dynamics among the channels is a task yet to be tackled.

\section{More Than Two Channels}

To study the problem of time-invariant systems with more than two channels, it is necessary to understand the concept of a strongly connected system [3]. A p-channel system is termed strongly connected if for every partition of the channels into disjoint sets $A$ and $B, C_{A}(s I-A)^{-1} B_{B}$ $\neq 0$. Equivalently, there can be no ordering of the channels for which the system transfer function matrix is block triangular. Equivalently again, there must be a path between every two nodes of the system graph (this idea is explained in the Appendix).

In [3], it is explained that any time-invariant system can be represented as a collection of strongly connected subsystems which can have only one-way connections between them. Moreover, all questions of decentralized control, observing, etc., can be analyzed by considering the individual strongly connected subsystems, each described by minimal state-variable realizations, together with any modes in the overall system description which are not included in the union of the modes of minimal descriptions of the strongly connected subsystems. These conclusions apply for all linear time-invariant feedback controllers, so long as the decentralized constraint is maintained.

We now consider the variation to these ideas required when periodic gains are present.

The definition of strong connectivity for time-invariant systems requires that certain transfer function matrices be nonzero, and in this sense is inappropriate for time-varying 
systems. For the purposes of this paper only, we extend the definition to encompass a special class of systems with periodically varying, piecewise constant matrices in the system equations. We require that where in the definition of connectivity for the time-invariant case a transfer function matrix is not identically zero, the corresponding collection of transfer function matrices computable from all the frozen values of the matrices in the system equations not be identically zero. For example, if in the time-invariant case, there is the connectivity condition $C(s I-A)^{-1} B \neq 0$, and if $A$ is replaced by a periodically time-varying $A(t)$, taking just two constant values $A_{1}$ and $A_{2}$, then we require in this paper that $C\left(s I-A_{1}\right)^{-1} B Z 0$ and $C\left(s I-A_{2}\right) B Z 0$.

We shall need the following result, the proof of which is contained in the Appendix.

Theorem 3.1: Consider a $p$-channel strongly connected system, and suppose a $(p-1)$-channel system is formed by putting feedback of the form $u_{p}=-K_{p} y_{p}$ around the $p$ th channel. Here $K_{p}$ is constant or piecewise constant. Then the resulting $(p-1)$-channel system is, for generic $K_{p}$, strongly connected.

We remark that the result is actually true for more complicated (e.g., dynamic) feedback. We shall, however, only need the present form.

It is straightforward to verify that if a system with periodic time-varying gains is not strongly connected, it can be decomposed into a collection of strongly connected subsystems which can only have one way connections between them and that, as for the time-invariant case, decentralized control questions must be analyzed by considering the individual subsystems. Accordingly, to explain the main ideas of the section, we shall confine attention to a three channel, strongly connected system.

Suppose we aim to use feedback on channels two and three to provide (uniform) controllability and observability at input and output one. Temporariliy consider channels one and two together as a single channel $\boldsymbol{A}$. It is immediately clear that unless using channel $A$ one can observe and control the system with a feedback gain around channel three, there is no possibility of doing the same with channel one, given feedback round channels two and three.

Using the ideas of Section II, we see observability and controllability from channel $\boldsymbol{A}$ can be achieved by feedback round channel three; in case there are no fixed modes, this feedback round channel three can be constant, and almost any constant feedback gain suffices. If, however, there is a $\lambda$ such that

$$
\operatorname{rank}\left[\begin{array}{ccc}
\lambda I-A & B_{1} & B_{2} \\
C_{3} & 0 & 0
\end{array}\right]<n
$$

or

$$
\operatorname{rank}\left[\begin{array}{cc}
\lambda I-A & B_{3} \\
C_{1} & 0 \\
C_{2} & 0
\end{array}\right]<n
$$

a constant gain will not suffice, but a piecewise constant periodic gain taking at least $\rho_{3}$ different values $\left[\rho_{3}=1+\right.$ $\left.\max \left(\operatorname{dim} u_{3}, \operatorname{dim} y_{3}\right)\right]$ will suffice.
With this feedback, there now results a two-channel system, possibly periodically time-varying, which is uniformly controllable and observable. By virtue of Theorem 3.1 , it is, at least for generic periodic or constant gains around channel three, strongly connected. The question arises as to whether we can now apply feedback round channel two to make the system uniformly controllable and observable from channel one. The answer is yes; we shall argue simply the observability.

If this two-channel system is time-invariant, the result is immediate by the results of Section II. So suppose that it is described by $\left\{A(t),\left[\begin{array}{ll}B_{1} & B_{2}\end{array}\right],\left[\begin{array}{ll}C_{1}^{\prime} & C_{2}^{\prime}\end{array}\right]^{\prime}\right\}$ where $A(t)$ is periodic and piecewise constant. Let us assume that $A(t)$ in fact takes the value $\bar{A}$ in $\left[s, s+T_{1}\right), \tilde{A}$ in $\left[s+T_{1}, s+T\right)$. Observability means that if there exists $\gamma$ for which

$$
\left[\begin{array}{l}
C_{1} \\
C_{2}
\end{array}\right] e^{\bar{A} t} \gamma=0 \quad\left[\begin{array}{l}
C_{1} \\
\dot{C}_{2}
\end{array}\right] e^{\tilde{A} t} e^{\bar{A} T_{1}} \gamma=0
$$

then $\gamma=0$. [This can be checked by examining the observability Grammian over $(s, s+T)$ ]. Equivalently, (take $\delta=$ $\left.e^{\bar{A} T_{1}} \gamma\right)$, the equations

$$
\left[\begin{array}{l}
C_{1} \\
C_{2}
\end{array}\right] e^{\bar{A} t} \delta=0 \quad\left[\begin{array}{l}
C_{1} \\
C_{2}
\end{array}\right] e^{\tilde{A} t} \delta=0
$$

imply $\delta=0$. If the "frozen" systems $\left\{\bar{A},\left[B_{1}, B_{2}\right],\left[C_{1}^{\prime}\right.\right.$ $\left.\left.C_{2}^{\prime}\right]^{\prime}\right\}$ and $\left\{\tilde{A},\left[\begin{array}{ll}B_{1} & B_{2}\end{array}\right],\left[\begin{array}{ll}C_{1}^{\prime} & C_{2}^{\prime}\end{array}\right]^{\prime}\right\}$ were to have no fixed modes (other than any associated with lack of centralized controllability and observability), then constant feedback around channel two would generically produce uniform controllability and observability at channel one. However, it is obvious from the definition of fixed modes that if the original three-channel system has fixed modes, so must each of the frozen two-channel systems. We now explain what is done in this case.

For convenience, suppose that $y_{2}$ is a scalar. We then take $u_{2}=K_{2}(t) y_{2}$ where $K_{2}(t)=0, t \in\left[s, s_{1}\right), K_{2}(t)=\bar{K}_{2}$, $t \in\left[s_{1}, s+T_{1}\right), K_{2}(t)=0, t \in\left[s+T_{1} s_{2}\right), K_{2}(t)=\tilde{K}_{2}, t \in\left[s_{2}, s\right.$ $+T)$ with $K_{2}(t)$ periodic. If $\gamma_{1}$ is a null vector of the observability Grammian over $[s, s+T]$ of $[A(t)+$ $\left.B_{2} K_{2}(t) C_{2}, C_{1}\right]$, then

$$
\begin{aligned}
& C_{1} e^{\overline{A t}} \gamma_{1}=0 \\
& C_{1} e^{\left(\bar{A}+B_{2} \bar{K}_{2} C_{2}\right) r} e^{\bar{A} s_{1}} \gamma_{1}=0 \\
& C_{1} e^{\tilde{A} t} e^{\left(A+B_{2} \bar{K}_{2} C_{2}\right)\left(T_{1}+s-s_{1}\right)} e^{\bar{A} s_{1}} \gamma_{1}=0 \\
& C_{1} e^{\left(\tilde{A}+B_{2} \tilde{K_{2}} C_{2}\right) r} e^{\tilde{A\left(s_{2}-s-T_{1}\right)}} \\
& \cdot e^{\left(A+B_{2} \bar{K}_{2} C_{2}\right)\left(T_{1}+s-s_{1}\right)} e^{A s_{1}} \gamma_{1}=0 .
\end{aligned}
$$

Arguing as in the last section, and using the fact that $C_{1}(s I-\bar{A})^{-1} B_{2} \neq 0$ in the light of strong connectivity, we conclude from the first two equations that

$$
C_{1} e^{\overline{A t}} \gamma_{1}=0 \quad C_{2} e^{\overline{A t}} \gamma_{1}=0
$$

for all $t$, and in fact if $\delta=e^{\left(\bar{A}+B_{2} \bar{K}_{2} C_{2}\right)\left(T_{1}+s-s_{1}\right)} e^{\bar{A} s_{1}} \gamma_{1}$, then

$$
\text { - } \quad C_{1} e^{\bar{A} t} \delta=0 \quad C_{2} e^{\bar{A} t} \delta=0 .
$$

In a similar manner the last two equations in (3.1) yield

$$
C_{1} e^{\tilde{A} t} \delta=0 \quad C_{2} e^{\tilde{A} t} \delta=0 .
$$


However, as argued above, the observability of the twochannel system implies that in (3.2) and (3.3) we have $\delta=0$, and thus, $\gamma_{1}=0$, i.e., the single-channel system is observable.

The above analysis applied for scalar $y_{2}$. The technique of Section II can be used to derive the result for vector $y_{2}$.

The procedure for coping with a $p$-channel system when $p>3$ is a straightforward extension of the procedure for a three-channel system. Assuming the $p$-channel system is strongly connected and meets a centralized controllability and observability condition, one successively applies feedback round channels $p, p-1, \cdots, 2$. The feedback can be constant only if there are no fixed modes associated with any of the frozen systems encountered at any stage in the procedure. Otherwise, it must be periodic and piecewise constant, taking a certain minimum number of values that is readily computable at each stage. The end result is that for generic values of all the feedback gains, the one-channel system is uniformly controllable and observable.

Though we do not show it here, we remark that if there are no decentralized fixed modes for the $p$-channel system, a generic selection of the constant feedback gain round channel $p$ will ensure that the resulting $(p-1)$-channel system has no fixed modes. Conversely, as is obvious from the definition, if the $p$-channel system has decentralized fixed modes, the $(p-1)$-channel system obtained via periodic feedback round channel $p$ for each frozen value of the feedback has a fixed mode.

\section{Conclusions}

Results for decentralized control problems have typically relied on centralized controllability and observability, on certain graph theoretic properties such as strong connectivity, and on freedom from decentralized fixed modes. The contribution of this paper has been to show that the presence of fixed modes need not prevent many results holding-provided one is prepared to widen the class of controllers considered to being periodically time-varying.

This means that there are indeed some linear timeinvariant system where satisfactory decentralized control can only be achieved when linear time-varying controllers are used.

\section{APPENDIX}

Graph theoretic discussion of strong connectivity. With each channel of a $p$-channel system, associate a node of a $p$-node graph, and draw a directed arc connecting node $i$ to node $j$ just in case $C_{j}(s I-A)^{-1} B_{i} \neq 0$, where in the periodically varying, piecewise constant situation, this inequality is understood to hold for all values of the relevant matrices.

A path from node $j_{1}$ to $j_{r}$ is a set of nodes $j_{1} j_{2}, \cdots j_{r}$ such that there is an arc from $j_{i}$ to $j_{l+1}, i=1, \cdots r-1$. The intuition is that if there is feedback from output to input of channel $j_{2}, \cdots, j_{r-1}$, then it will be possible for signals inserted at input $j_{1}$ to affect output $j_{r}$, even in the absence of a direct connection.
A system is termed strongly connected if there exists a path between any two nodes. Equivalent formulations of the strong connectivity property can be found in [3].

Preservation of connectivity given feedback round a channel. We now prove Theorem 3.1. Consider any two nodes $j_{1}, j_{r}$ of the graph associated with the $(p-1)$-channel system derived after introducing feedback to the original $p$-channel system. Before the introduction of this feedback, these two nodes, regarded as nodes of the graph of the $p$-channel system, define the end points of a path because the $p$-channel system is strongly connected. We distinguish the following cases.

Case 1: The path does not include node $p$.

Case 2: The path includes node $p$.

Let $W_{j i}$ denote $C_{j}(s I-A)^{-1} B_{i}$ (or the collection of such quantities), and $\frac{j}{W_{j i}}$ denote the corresponding quantity resulting after feedback. Under case one, we have $W_{j_{2} j_{1}} \not$ $0, \cdots, W_{j_{r} j_{r-1}} \not \geq 0$. Since for one specialized feedback, viz. $u_{p} \equiv 0$, we have $\bar{W}_{j_{2} j_{1}}=W_{j_{2} j_{1}} \not \geq 0, \cdots \bar{W}_{j_{s} j_{r-1}}=W_{j_{r} j_{r-1}} \not \geq 0$ it follows that for almost all feedback, i.e., generically, we must have $\bar{W}_{j_{2} j_{1}} \not 0, \cdots, \bar{W}_{j_{r} j_{r-1}} \not 0$, i.e., a path connects $j_{1}$ to $j_{r}$ for the $(p-1)$-channel system-the same path in effect as in the $p$-channel system.

Under case two, suppose the path is $j_{1}, j_{2}, \cdots$, $j_{k}, p, j_{k+2}, \cdots, j_{r}$. Arguing as for case one, we know that generically, $\quad \bar{W}_{j_{2} j_{1}} \not \geq 0, \cdots, \bar{W}_{j_{k} j_{k-1}} \not 0, \quad \bar{W}_{j_{k-3} j_{k-2}} \not$ $0, \cdots \bar{W}_{j_{r} j_{r},} \neq 0$. We must show that generically $\bar{W}_{j_{k-2} j_{k}} \neq 0$. If $W_{j_{k+j_{k}}} \neq 0$, we can apply the case one argument. So assume that $W_{j_{k+3} j_{k}} \equiv 0$. Then

$$
\bar{W}_{j_{k+2} j_{k}}=-W_{j_{k+2} p} K_{p}\left(I+W_{p p} K_{p}\right)^{-1} W_{p j_{k}}
$$

as an easy calculation shows. Since $W_{j_{k+2} p} \neq 0, W_{p j_{k}} \neq 0$, and $K_{p}$ are arbitrary, we have for generic $K_{p}$ that $\bar{W}_{j_{k+2} j_{k}} \neq 0$. Consequently, in the graph of the $(p-1)$-channel system, there is a path $j_{1}, \cdots, j_{k}, j_{k+2}, \cdots, j_{r}$ connecting nodes $j_{1}$ to $j_{r}$. This establishes the strong connectivity result.

\section{REFERENCES}

[1] M. Aoki, "On feedback stabilizability of decentralized dynamic systems," Automatica, vol. 8, pp. 163-173, 1972.

[2] J. P. Corfmat and A. S. Morse, "Control of linear systems through specified input channels." SIAM J. Contr. Optimiz., vol. 14, pp. 163-175, Jan. 1976

[3] - - "Decentralized control of linear multivariable systems," Automatica. vol. 12, pp. 479-495, 1976.

[3] E. J. Davison and S. H. Wang, "Properties of linear time-invariant multivariable systems subject to arbitrary output and state feedback." IEEE Trans. Automat. Contr., vol. AC-18, pp. 24-32, 1973.

[5] S. H. Wang and D. J. Davison, "On the stabilization of decentralized control systems," IEEE Trans. Automat. Contr., vol. AC-18, pp. $473-478,1973$

[6] B. D. O. Anderson and D. J. Clements, "Algebraic characterization of fixed modes in decentralized control," Automatica. Sept. 1981, to appear.

[7] H. Kobayashi, H. Hanafusa, and T. Yoshikawa, "Controllability under decentralized information structure," IEEE Trans. Automat. Contr., vol. AC-23, pp. 182-188, Apr. 1978.

[8] J. M. Potter, B. D. O. Anderson, and A. S. Morse. "Single channel control of a two-channel system." IEEE Trans. Automat. Contr., vol. AC-24, pp. 491-492. June 1979.

[9] L. M. Silverman and B. D. O. Anderson, "Controllability, observability and stability of linear systems," SIAM J. Contr., vol. 6. pp. 121-130. Feb. 1968.

[10] K. Kendig, Elementary Algebraic Geometry. New York: SpringerVerlag. 1977. 


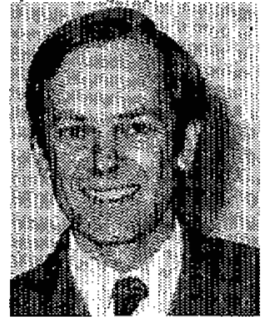

Brian D. O. Anderson (S'62-M'66-SM'74-F'75) was born in Sydney, Australia in 1941. He received the B.S. degree in pure mathematics and electrical engineering from the University of Sydney, Sydney, Australia, and the Ph.D. degree in electrical engineering from Stanford University, Stanford, CA.

Before joining the University of Newcastle in 1967, where he is a Professor in the Department of Electrical Engineering, he was a faculty member of the Department of Electrical Engineering at Stanford University and a Staff Consultant at Vidar Corporation, Mountain View, CA. He has also held appointments as a Visiting Professor at Southern Methodist University, University of Massachusetts, University of California at Berkeley, and Stanford University. He was Head of the Department of Electrical Engineering at the University of Newcastle from 1967 to 1975. His interests are in control, communication systems, and networks, and he has written many papers in these areas. $\mathrm{He}$ is coauthor of five books, Linear Optimal Control, Network Analysis and Synthesis, Foundation of Systems Theory, and most recently Singular Optimal Control, with D. J. Clements, and Optimal Filtering, with J. B. Moore. From 1971 to 1977, he was a member of the Australian Research Grants Committee and since 1977 has been a member of the Australian Science and Technology Council.
Dr. Anderson is a Fellow of the Australian Academy of Science, Australian Academy of Technological Sciences and Institution of Engineers, Australia, and a member of Sigma Xi, SLAM and Eta Kappa Nu.

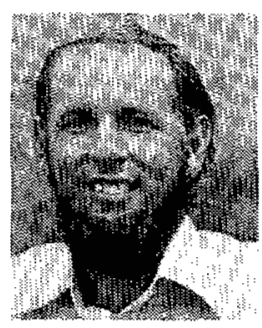

John B. Moore (S'66-M'67-SM'77-F'79) was born in China in 1941: He received the B.S., M.S., and $\mathrm{Ph} . \mathrm{D}$. degrees in electrical engineering from the University of Santa Clara, Santa Clara, CA in 1963,1964, and 1967, respectively.

He was appointed Senior Lecturer at the University of Newcastle in 1967, promoted to Associate Professor in 1968 and Full Professor (personal chair) in 1973. He has held other visiting academic appointments at the University of Santa Clara in 1968, at the University of Maryland in 1970, Colorado State University and Imperial College in 1974, and at University of California at Davis in 1977. He has spent periods in industry as a design engineer and as a consultant. His current research in control and communication systems is supported by the Australian Research Grants Committee. He is co-author with B. D. O. Anderson of two books Linear Optimal Control and Optimal Filtering (Englewood Cliffs, NJ: Prentice-Hall, 1971 and 1979, respectively).

\title{
Continuous State Feedback Guaranteeing Uniform Ultimate Boundedness for Uncertain Dynamic Systems
}

\author{
MARTIN J. CORLESS, STUDENT MEMBER, IEEE, AND GEORGE LEITMANN
}

\begin{abstract}
We consider a dynamic system containing uncertain elements. Only the set of possible values of these uncertainties is known. Based on this information a class of state feedback controls is proposed in order to guarantee uniform ultimate boundedness of every system response within an arbitrarily small neighborhood of the zero state. These feedback controls are continuous functions of the state.
\end{abstract}

\section{INTRODUCTION}

$\mathrm{T}$ HE PROBLEM of designing a state feedback control that guarantees the desired performance of a dynamic system containing uncertain elements is discussed in [1]-[6], among others. The desired performance is usually uniform

Manuscript received April 13, 1981. This work was supported by the National Science Foundation under Grant ENG 78-13931.

The authors are with the Department of Mechanical Engineering, University of California, Berkeley, CA 94720. asymptotic stability of an equilibrium state [1]-[4]. Sometimes one is content with uniform ultimate boundedness in some set [5],[6]; in that case one can consider feedback based on uncertain state or output [5]. The salient feature of the problem is the fact that it is a deterministic treatment of uncertainty in that one requires certain performance in the presence of uncertain information. The essential knowledge about the uncertain elements concerns only their possible size; that is, only the sets in which the values of the uncertain quantities can range are presumed to be known.

If some conditions are satisfied-primary among which are the so-called "matching conditions"] (see [1]-[6]) - then all uncertain elements can be "lumped" and the system is described by

\footnotetext{
${ }^{\mathrm{I}}$ These can be relaxed (see [7]).
} 